\title{
Rolle der 24-Stunden-Augeninnendruckmessung in der Glaukombehandlung
}

\author{
Chun Hing $\mathrm{Ho}^{\mathrm{a}}$ Jasper K. W. Wong ${ }^{\mathrm{b}}$ \\ aLi Ka Shing Faculty of Medicine, The University of Hong Kong, Hong Kong, Hong Kong; \\ ${ }^{b}$ Department of Ophthalmology, Li Ka Shing Faculty of Medicine, The University of Hong Kong, Hong Kong, Hong Kong
}

\section{Zusammenfassung}

Das Glaukom ist weltweit die häufigste Ursache für irreversible Erblindung, und die Prävalenz steigt. Grundpfeiler der Behandlung ist die Senkung des Augeninnendrucks (Intraokulardruck, IOD). Derzeit basiert das IOD-Monitoring auf punktuellen Messungen in der Praxis. Bei bis zu 50\% der Glaukompatienten war der initiale IOD jedoch normal, während einige behandelte Patienten trotz niedrigem IOD progrediente glaukomatöse Sehnervschädigungen aufwiesen. Kürzlich veröffentlichte Studien zeigten, dass der IOD von Glaukompatienten im Tagesverlauf mit unterschiedlichen Mustern fluktuiert, und bei einigen traten die IOD-Peaks außerhalb der Praxiszeiten auf. Diese Ergebnisse brachten neue Erkenntnisse über die Rolle der 24-Stunden-IOD-Messung in der Behandlung des Normaldruckglaukoms und von Patienten mit progredienter Verschlechterung trotz scheinbar gut kontrolliertem IOD. Allerdings sind die bislang vorliegenden Ergebnisse recht uneinheitlich, und es besteht noch kein Konsens. In der vorliegenden Übersichtsarbeit werden die derzeitigen Modalitäten der 24-Stunden-IOD-Messung kurz erläutert und das charakteristische 24-Stunden-IOD-Profil sowie die klinische Bedeutung der IOD-Parameter für die Vorhersage der Glaukomprogression bei verschiedenen Glaukom-Subtypen zusammengefasst. Darüber hinaus wird die Wirksamkeit der derzeitigen Therapiemodalitäten zur Glaukombehandlung in Bezug auf die beschriebenen 24-Stunden-IOD-Profile erörtert. Ziel ist es, die Rolle der 24-Stunden-IOD-Messung bei der Ermittlung und Stratifizierung des Progressionsrisikos von Glaukompatienten zu stärken und die Therapie entsprechend den IOD-Profilen weiter zu verbessern.

c) 2019 Chun Hing Ho and Jasper K. W. Wong

\section{Einleitung}

Definitionsgemäß handelt es sich beim Glaukom um eine Gruppe von fortschreitenden Opticus-Neuropathien, die durch eine Degeneration der retinalen Ganglienzellen und dadurch bedingte Veränderungen des Sehnervenkopfs gekennzeichnet sind und die im weiteren Verlauf zu Gesichtsfeldausfällen führen [1, 2]. Das Glaukom ist weltweit die häufigste Ursache für irreversible Erblindung [3]. Die weltweite Prävalenz des Glaukoms bei den 40 bis 80 -Jährigen liegt bei 3,54\%, und Schätzungen zufolge wird die Zahl der Betroffenen auf 76 Millionen im Jahr 2020 und 111,8 Millionen im Jahr 2040 ansteigen [4].

Ein erhöhter Augeninnendruck (IOD) gilt insbesondere als Hauptrisikofaktor für die Glaukomprogression [5], die sich durch eine Verdünnung der retinalen Nervenfaserschicht (retinal nerve fibre layer, RNFL) [6] und Gesichtsfeldverschlechterung [1,2] äu-
Bert. Hoher IOD verursacht eine mechanische Spannung und Belastung der posterioren Augenstrukturen, besonders der Lamina cribrosa, einem siebförmigen Abschnitt der Sklera, wo die retinalen Ganglienzellaxone das Auge verlassen [7]. Durch Kompression, Deformation und Remodeling der Lamina cribrosa, die den schwächsten Punkt des Auges bei IOD-induzierter Belastung bildet, kommt es beim Glaukom zu Störungen der axonalen Transports und zu neuronalen Schädigungen [2, 8].

Interessanterweise kann die glaukombedingte Opticus-Neuropathie auch bei Personen mit normalem IOD auftreten, beispielsweise beim Normaldruckglaukom (normal tension glaucoma, NTG) [2, 9-11] und das Glaukom kann auch bei Patienten mit zufriedenstellender IOD-Kontrolle weiter fortschreiten [11]. Demgegenüber entwickeln manche Menschen mit hohem IOD nie Sehnervenschäden, wie zum Beispiel bei okulärer Hypertension (OHT) [12]. Zur Erklärung dieses Phänomens wurden ver- information@karger.com

www.karger.com/kop

(c) 2020 S.Karger GmbH, Freiburg

Karger"
Jasper K. W. Wong

Department of Ophthalmology

Li Ka Shing Faculty of Medicine, The University of Hong Kong

6/F, William MW Mong Block, 21 Sassoon Road, Pokfulam, Hong Kong, Hong Kong jasperwongkw@gmail.com 
schiedene Mechanismen postuliert, darunter Schwankungen in der Mikrozirkulation des Sehnervenkopfes (optic nerve head, $\mathrm{ONH}$ ) [13-16] und Probleme bei der IOD-Messung.

Kürzlich durchgeführte Studien sprechen dafür, dass neben den punktuellen IOD-Messungen in der Praxis [1] auch die Peaks, der Verlauf und Schwankungen des IODs eine Rolle bei der Glaukomprogression spielen [1]. In einer Untersuchung von Park et al. mit Patienten mit juvenilem Offenwinkelglaukom (juvenile openangle glaucoma, JOAG) kam es bei den Patienten trotz optimaler medikamentöser Behandlung und scheinbar gut eingestelltem IOD zu temporärem Verschwommensehen oder fortschreitenden Gesichtsfeldausfällen. Bei diesen Patienten wurden im weiteren Verlauf stärkere IOD-Fluktuationen über den Tag festgestellt [11]. Ferner liegen Belege dafür vor, dass bei mehr als der Hälfte der Glaukompatienten die IOD-Peaks außerhalb der Praxiszeiten auftreten [17]. Eine retrospektive Übersichtsarbeit ergab außerdem, dass der tagsüber auftretende IOD-Peak durchschnittlich 4,9 mmHg höher war als der auf herkömmliche Weise in der Praxis gemessene IOD-Höchstwert [17]. In einer Studie, in der die 24-Stunden-IOD-Messung mit den herkömmlichen IOD-Messungen in der Praxis verglichen wurde, hatte die 24-StundenIOD-Messung bei 79,3\% der Patienten eine Therapieänderung zur Folge [18]. Diese Ergebnisse zeigen, dass die derzeitige Praxis der IOD-Messung in der Tat unzureichend ist und weitere Diskussionen rechtfertigt [19].

In der vorliegenden Übersichtsarbeit werden zunächst die derzeitigen Modalitäten der 24-Stunden-IOD-Messung kurz beschrieben. Außerdem werden die Muster der 24-Stunden-IOD-Messung bei verschiedenen Glaukomtypen erläutert und die Korrelation der verschiedenen IOD-Parameter mit der Glaukomprogression zusammengefasst. Darüber hinaus wird die Evidenzlage zur Wirksamkeit der derzeitigen Glaukom-Therapiemodalitäten in Bezug auf die untersuchten 24-Stunden-IOD-Profile dargestellt.

\section{Methode}

Es erfolgte eine Suche in der PubMed-Datenbank von deren Beginn bis zum 23. Juni 2019 mit Kombinationen der folgenden Suchbegriffe: «glaucoma», «intraocular pressure», «diurnal», «nocturnal», «circadian», «24-hour», «monitoring», «fluctuation», «progression» und «visual field» für randomisierte klinische Studien, Metaanalysen, systematische Übersichtsarbeiten sowie interventionelle Studien und Beobachtungsstudien. Eingeschlossen wurden Artikel über die 24-Stunden-IOD-Messung sowie die Therapiemodalitäten bei verschiedenen Glaukomtypen, die das Ergebnis einer umfangreichen Suche ohne Spracheinschränkungen waren.

\section{Modalitäten der 24-Stunden-IODmessung in der klinischen Praxis}

Im klinischen Alltag häufig eingesetzte Verfahren zur IOD-Messung sind die Goldmann Applanationstonometrie (GAT), die Applanationstonometrie mittels Handgerät und die Pneumotonometrie. Diese Methoden werden hauptsächlich für punktuelle
IOD-Messungen in der Praxis eingesetzt und nicht für die 24-Stunden-IOD-Messung, denn dies wäre relativ zeitaufwändig und unpraktisch [20]. Da der IOD dynamischer Natur ist und tageszeitlichen Veränderungen sowie täglichen Fluktuationen unterliegt, wurde in den vergangenen Jahrzehnten die Entwicklung von Messmethoden zur ambulanten 24-Stunden-IOD-Messung vorangetrieben. $\mathrm{Zu}$ diesen Verfahren gehören vor allem die Selbsttonometrie, die permanenteIOD-Messung und die24-Stunden-IOD-Messung.

Bei der Selbsttonometrie müssen die Patienten in regelmäßigen Abständen über 24 Stunden den eigenen IOD mit einem tragbaren Tonometer, z.B. dem Icare ONE Rebound-Tonometer (RTONE, Icare Finland Oy, Vantaa, Finnland), bestimmen. Sood und Ramanathan führten eine Studie durch, in der 18 Patienten mit behandeltem NTG ein Jahr lang das Icare ONE ReboundTonometer verwendeten [21]. Die mittels RTONE gemessenen IOD-Werte zeigten eine starke Korrelation mit den Ergebnissen des GAT, das bei Praxisvisiten verwendet wurde. Bei NTG-Patienten mit unverhältnismäßig starker Krankheitsprogression im Verhältnis zu den in der Praxis gemessenen IOD-Werten kann die Verwendung von RTONE helfen, höhere IOD-Druckspitzen zu erkennen, die durch Messungen in der Praxis nicht festgestellt wurden. Daher stellt die Selbsttonometrie definitiv eine wertvolle Ergänzung zu den punktuellen Messungen in der Praxis dar, da sie ein umfassenderes Bild der tagsüber auftretenden IOD-Fluktuationen liefert und so die Glaukombehandlung unterstützt [22]. Allerdings ist zu beachten, dass die Selbsttonometrie für ältere Glaukompatienten eine technische Herausforderung darstellt und damit auch keine IOD-Messungen im Schlaf durchgeführt werden können [23].

Die permanente IOD-Messung erfordert die operative Implantation eines IOD-Sensors. Bei diesem handelt es sich um einen telemetrischen Druckwandler oder einen in eine Intraokularlinse integrierten Drucksensor. Koutsonas et al. führten die erste prospektive klinische Studie zur telemetrischen intraokularen Drucküberwachung (ARGOS-Studie) durch. Ihren Angaben zufolge wurde der IOD-Sensor trotz einer möglichen in der postoperativen Frühphase auftretenden Vorderkammerentzündung und leichten bis mittelschweren Pupillenverziehung sowie Pigmentdispersion von allen Patienten gut vertragen [24]. Darüber hinaus führten Koutsonas et al. im Rahmen einer weiteren Machbarkeitsstudie mit 6 Patienten mit primärem Offenwinkelglaukom (primary open-angle glaucoma, POAG) [25] erfolgreich wiederholte und automatisierte 24-Stunden-IOD-Messungen mit einem Prototyp eines berührungsfreien Messsystems durch. Dabei berichteten sie keine schwerwiegenden Nebenwirkungen im Langzeit-Follow-up-Sicherheitsbericht [26]. Die permanente IODMessung mithilfe eines implantierten IOD-Sensors ist im Allgemeinen zuverlässiger und ermöglicht außerdem die Erfassung von 24-Stunden-Daten (einschließlich Daten während des Schlafes) über einen längeren Zeitraum zur Verlaufskontrolle sowie für klinische Studien. Dies erfordert jedoch einen vergleichsweise großen operativen Eingriff und es bestehen potenzielle Sicherheitsbedenken [23].
Kompass Ophthalmol 2020;6:5-14 DOI: $10.1159 / 000505890$ 
Ein von der US-amerikanischen Behörde für Lebensmittelüberwachung und Arzneimittelzulassung (Food and Drug Administration, FDA) zugelassenes System zur kontinuierlichen 24-Stunden-IOD-Messung ist der SENSIMED Triggerfish ${ }^{\circledR}$ Einweg-Kontaktlinsen-Sensor (Triggerfish CLS, Sensimed AG, Lausanne, Schweiz). Verschiedenen Studien zufolge war die mittels visueller Analogskala (wobei Null «Keine Beschwerden» und 100 «Sehr starke Beschwerden» bedeutete) gemessene Verträglichkeit zufriedenstellend und lag zwischen 21,82 und 27,2 [20, 27, 28]. Das Triggerfisch CLS-System ist besser als die beiden anderen genannten Methoden, da es nicht invasiv, leicht zu entfernen und breit verfügbar ist [23]. Darüber hinaus ist es in der Lage, IODFluktuationen über bis zu 24 Stunden, einschließlich der Stunden während des ungestörten Nachtschlafs, zu messen [23]. Zudem zeigte das Triggerfish CLS-System in Untersuchungen von Mansouri et al. [28], Mottet et al. [29] und Holló et al. [30] eine hohe Reproduzierbarkeit. Die Validität des CLS-Systems ist jedoch nicht bekannt. Möglicherweise hat dies damit zu tun, dass das CLS-System den IOD nicht direkt misst, sondern die IODSchwankungen anhand der gemessenen Krümmungsänderungen des kornealen Limbus abschätzt [29, 30]. In aktuellen Studien bestand eine hohe Korrelation zwischen den mittels GAT und CLS gemessenen IOD-Werten zum Zeitpunkt der CLS-Einbringung [31], wohingegen die Korrelation 24 Stunden nach der CLSEinbringung gering ausfiel $[29,30]$. Diese Abweichung könnte dadurch bedingt sein, dass die CLS-Kurven mit zunehmender CLSTragedauer einen Trend zu steigenden Messwerten aufwiesen [30]. Zudem treten beim CLS-System häufig Nebenwirkungen auf wie Verschwommensehen, konjunktivale Hyperämie und Keratitis superficialis punctata, die allgemein als leicht eingestuft werden und sich innerhalb von 48 Stunden zurückbilden [28].

\section{Charakteristische 24-Stunden-IOD-Muster und ihr prädiktiver Wert für die Progression verschiedener Glaukomtypen}

Gesunde haben einen mittleren IOD von $14,7 \pm 2,8 \mathrm{mmHg}$ [32], mit kurzzeitigen IOD-Fluktuationen von etwa $4-6 \mathrm{mmHg}$ [9]. Morgens und am frühen Nachmittag ist der IOD typischerweise am höchsten, während die Tiefstwerte am Nachmittag und nachts auftreten [6, 19, 33]; zudem kann es eine mögliche IOD-Druckspitze beim Aufwachen geben [34].

Glaukompatienten weisen dagegen im Allgemeinen höhere Druckspitzen in der 24-Stunden-IOD-Messung auf, und es dauert länger, bis diese abgeklungen sind [34]. Mit 6-15 mmHg fallen die kurzzeitigen IOD-Fluktuationen bei Glaukompatienten signifikant stärker aus als bei gesunden Probanden. Im Extremfall können die IOD-Fluktuationen sogar bis zu $40 \mathrm{mmHg}$ betragen [19, 35-37]. Die tagsüber auftretenden IOD-Fluktuationen liegen bei Glaukompatienten zwischen 4,8-11 $\mathrm{mmHg}$, gegenüber nur 3,17-5 mmHg bei gesunden Probanden [38]. Eine Studie an 296 Augen von 213 Patienten mit primärem Offenwinkelglaukom und primärem Engwinkelglaukom (primary angle closure glaucoma, PACG) berichtete eine Schwankung über Tag von $>6$
mmHg bei einem IOD von $\geq 21 \mathrm{mmHg}$, die bei gesunden Augen nicht nachweisbar war [39]. Zudem wurden bei Glaukompatienten häufigere Peaks über Tag und in der Nacht festgestellt [40, 41]. In etlichen Studien wurde der Versuch unternommen, die Daten der Advanced Glaucoma Intervention Study (AGIS) zu analysieren. Nouri-Mahdavi et al. zufolge sind IOD-Fluktuationen ein signifikanter Risikofaktor für die Gesichtsfeldprogression. Diese Studie schloss allerdings auch Daten ein, die nach der Therapie erhoben wurden, was möglicherweise zu den IOD-Fluktuationen bei Patienten mit fortgeschrittener Gesichtsfeldeinschränkung beigetragen hat [43]. Caprioli und Coleman beschränkten sich bei ihrer Datenanalyse auf den Zeitraum vor der Gesichtsfeldprogression und schlossen operativ behandelte Patienten aus. Sie stellten fest, dass die IOD-Fluktuationen nur im unteren Terzil des mittleren IODs signifikant mit einer Gesichtsfeldprogression assoziiert waren, und sie waren der Ansicht, dass die IOD-Fluktuationen nur bei niedrigem IOD einen stärkeren Einfluss auf die Gesichtsfeldprogression haben; bei einem hohen IOD ist dagegen der mittlere IOD der Hauptrisikofaktor [43-46]. Andere Studien, in denen die AGIS- $[1,42]$ und die Glaukom-Progressionsstudie (GPS) analysiert wurden [1, 40, 47], kamen zu dem Ergebnis, dass eine höhere Anzahl von tagsüber auftretenden Peaks und langanhaltenden Peaks $[1,48]$ sowie eine höhere Peak-Latenz (kürzere Zeitabstände zwischen den Druckspitzen) ebenfalls mit einer schnelleren Gesichtsfeldabnahme verbunden waren. Demgegenüber wurde in einigen retrospektiven Analysen der Early Manifest Glaucoma Treatment Trial (EMGT) [49] und der Ocular Hypertension Treatment Study (OHTS) [50] keine Assoziation zwischen IOD-Fluktuationen und Glaukomprogression festgestellt [51]. Einige andere Studien wie die European Glaucoma Prevention Study (EGPS), die Diagnostic Innovations in Glaucoma Study (DIGS) [52, 53], eine Studie von Jonas und Mitarbeitern [19, 54] und eine klinische Studie von Fogagnolo et al. [55] fanden ebenfalls keinen statistisch signifikanten Einfluss der IOD-Fluktuationen auf die Glaukomprogression. Allerdings wurden in einigen der genannten Studien, wie beispielsweise in der OHTS, keine 24-Stunden-IOD-Daten erhoben und die IOD-Fluktuationen wurden nur als IOD-Standardabweichung im Rahmen der klinischen Visiten berechnet [51]. In manchen Studien erhielten die Patienten unterschiedliche antiglaukomatöse Arzneimittel, die den intrinsischen zirkadianen Rhythmus des IODs bei Glaukom verändern können. Vor dem Hintergrund, dass die verschiedenen Glaukomstudien, die unterschiedliche Glaukom-Subtypen einschlossen und die durchgeführt wurden, um die Bedeutung der 24-Stunden-IOD-Muster für die Glaukombehandlung besser zu verstehen, zu sehr unterschiedlichen Ergebnissen kamen, werden in der vorliegenden Arbeit die spezifischen 24-Stunden-IODMuster beschrieben und die jeweiligen Parameter zur Vorhersage der Glaukomprogression bei den verschiedenen Glaukomtypen zusammengefasst.

\section{Primäres und sekundäres Offenwinkelglaukom}

Das primäre Offenwinkelglaukom (POAG) ist die häufigste Form des Glaukoms [56]. In einer Studie mit 102 Probanden, die mit- 
hilfe eines berührungsfreien Tonometers alle 2 Stunden von 8.00 Uhr bis 24.00 Uhr IOD-Messungen vornahmen, betrug der mittlere IOD 19,9 $\pm 4,3 \mathrm{mmHg}$ [57] und in einer anderen Studie mit 64 POAG-Patienten lag er bei 17,6 $\pm 3,2 \mathrm{mmHg}$ [58]. Beide Ergebnisse fielen signifikant höher aus als bei gesunden Probanden, bei denen der Wert gemäß der Beijing Eye Study nur 14,7 $\pm 2,8 \mathrm{mmHg}$ betrug [32]. Das 24-Stunden-IOD-Muster bei POAG zeigt morgens die höchsten IOD-Werte [19,59]; diese nehmen im Laufe des Tages allmählich ab und sind um Mitternacht am niedrigsten [17]. Damit entsprechen sie dem Verlauf bei gesunden Probanden. In einer Studie von Xiao et al. trat der IOD-Peak bei 73,5\% der Patienten in der POAG-Gruppe und bei 59,6\% der gesunden Kontrollgruppe außerhalb der Praxiszeiten auf, typischerweise zwischen Mitternacht und 6 Uhr [57], und der IOD-Peak konnte bis zu 25,3 $\pm 5,6 \mathrm{mmHg}$ erreichen [57]. Bedingt durch die ausgeprägten täglichen Veränderungen fielen die gemessenen kurzzeitigen IODFluktuationen viel höher aus als bei gesunden Probanden und reichten von $8,31 \pm 2,58 \mathrm{mmHg}[6,19,39]$ bis $9,1 \pm 3,6 \mathrm{mmHg}$ [57]. Hughes et al. berichteten in einer retrospektiven Übersichtsarbeit mit 29 behandelten POAG- und NTG-Patienten, dass die IODPeaks während der 24-Stunden-Messung um fast $5 \mathrm{mmHg}$ höher ausfallen können als die in der Praxis gemessenen Peaks, und zirkadiane Messungen hatten bei bis zu 80\% der Patienten eine Therapieänderung zur Folge [18, 33].

Beim sekundären Offenwinkelglaukom wie dem Pigmentglaukom und dem exfoliativen Glaukom (XFG), bei dem es zu einer in Abständen auftretenden Ablagerung von Pigmenten und Exfoliationsmaterial im Trabekelmaschenwerk kommt, sind die 24-Stunden-IOD-Profile in der Regel variabler. Tojo et al. berichteten in einer Studie mit Patienten mit Pseudoexfoliationssyndrom über einen mittleren IOD von 20,3 $\pm 3,9$ mmHg [60]. Konstas et al. stellten fest, dass der IOD-Peak bei bis zu $45 \%$ der Patienten mit unbehandeltem XFG außerhalb der Praxiszeiten auftrat $[33,36]$. Nach Angaben von Jonas et al. lag der Druckgipfel bei Patienten mit sekundärem Offenwinkelglaukom am Nachmittag, wohingegen der Peak bei POAG-Patienten und gesunden Probanden morgens auftrat $[44,61]$. Das sekundäre Offenwinkelglaukom zeigte außerdem eine höhere IOD-Variabilität im Vergleich zur Kontrollgruppe und den POAG-Patienten [44, 61]. Patienten mit XFG weisen typischerweise stärkere kurzzeitige IOD-Fluktuationen sowie höhere IOD-Höchst- und Tiefstwerte auf als POAG-Patienten [19, 36, $62]$. Eine klinische Studie von Konstas et al. ergab kurzzeitige IODFluktuationen von > $15 \mathrm{mmHg}$ bei $35 \%$ der Patienten mit XFG, jedoch lediglich bei 7,5\% der POAG-Patienten [19, 36].

Die klinische Bedeutung des 24-Stunden-IOD-Musters bei POAG liegt in seinem prädiktiven Wert für die Krankheitsprogression. In einer klinischen Studie von Asrani et al. betrugen die jeweiligen Hazard Ratios der Kurz- und Langzeit-Fluktuationen des IODs für die Glaukomprogression 5,69 (95\%-Konfidenzintervall (KI): 1,86-17,35) bzw. 5,76 (95\%-KI: 2,21-14,98) nach Anpassung für den in der Praxis gemessenen IOD-Wert, Alter, ethnische Zugehörigkeit, Geschlecht und Gesichtsfeldeinschränkung bei Studienbeginn [58]. Die Korrelation zwischen den Kurz- und Langzeit-Fluktuationen des IODs und der Gesichtsfeldprogression bei
POAG wurde auch in anderen Studien von Asrani et al. [19, 33, 43, 44, 58], Naito et al. [63], Bergeå et al. [51, 64] sowie Rao et al. [65] nachgewiesen. De Moraes et al. zeigten in einer Querschnittsstudie, dass die Anzahl der langanhaltenden Peaks und das mittlere Peak-Verhältnis, die zu den kurzzeitigen IOD-Fluktuationen beitragen, ebenfalls gute Prädiktoren für die Glaukomprogression bei POAG-Patienten sind $[1,48]$. Beim XFG korrelieren nach einem Bericht von Bergeå et al. sowohl der mittlere IOD als auch die IOD-Fluktuationen mit der Gesichtsfeldprogression [43, 64]. Die Korrelation anderer IOD-Parameter mit der Glaukomprogression bei POAG-Patienten ist jedoch nicht eindeutig. Rao et al. fanden in ihrer Studie an 296 Augen von 213 POAG- und PACGPatienten [65] keine signifikante Korrelation zwischen dem mittleren IOD, dem IOD-Peak und der Geschwindigkeit der Gesichtsfeldprogression.

\section{Normaldruckglaukom}

Nach Pajic et al. [67] lag der mittlere IOD beim NTG zwischen $11,5 \pm 2,4 \mathrm{mmHg}$ [66] und 17,8 $\pm 1,6 \mathrm{mmHg}$ und fiel damit ähnlich aus wie bei gesunden Probanden. Quaranta et al. stellten in einer Studie mit 62 NTG-Patienten fest, dass 12,9\% der Patienten bei Messung in der gewohnten Position eine Akrophase am Tag aufwiesen, während $67,7 \%$ eine nächtliche Akrophase aufwiesen, und sich damit vom typischen Verlauf bei gesunden Probanden unterschieden [68]. In einer anderen Studie von Renard et al. zeigten NTG-Patienten zudem tagsüber oder in der Nacht auftretende Druckspitzen (54,5\% bzw. 36,4\%) [69]. Solche nächtlichen Druckspitzen können durch IOD-Messungen in der Praxis nicht erfasst werden $[66,70]$. Gleichwohl betrugen die am Tag auftretenden IOD-Fluktuationen bei NTG-Patienten Berichten zufolge 4,4 \pm $1,6 \mathrm{mmHg}$ [6] und unterschieden sich damit nicht signifikant von gesunden Probanden.

Choi et al. führten eine krankenhausbasierte Studie zur 24-Stunden-IOD-Messung mit 113 Patienten mit unbehandeltem NTG durch. In der retrospektiven Überprüfung dieser NTG-Patienten fand sich ein Zusammenhang zwischen einer raschen Glaukomprogression und den Langzeitfluktuationen des IODs [40, 51, 71]. Eine Studie mit 140 NTG-Patienten ergab außerdem, dass Patienten mit ausgeprägten Langzeitfluktuationen des IODs eine 5,26fach höhere Wahrscheinlichkeit für eine Glaukomprogression aufwiesen [6], was für einen starken Zusammenhang zwischen Langzeitfluktuationen des IODs und der Glaukomprogression bei NTG-Patienten spricht. Jonas et al. führten eine retrospektive Überprüfung der Krankenakten durch, die 855 Augen von 458 Patienten umfasste. Diese ergab, dass der mittlere IOD und der IOD-Tiefstwert ebenfalls unabhängige Prädiktoren für die Glaukomprogression bei NTG-Patienten waren [33, 54].

\section{Primäres Engwinkelglaukom}

In einer retrospektiven Analyse von Suresh et al. betrug der mittlere IOD bei Patienten mit PACG vor der Behandlung 29,9-45,8 $\mathrm{mmHg}$ [72]. Der zirkadiane IOD-Rhythmus bei PACG-Patienten glich dem bei POAG-Patienten und gesunden Probanden, mit der Ausnahme, dass Patienten mit PACG durchweg einen höheren
Kompass Ophthalmol 2020;6:5-14 DOI: $10.1159 / 000505890$ 
IOD aufwiesen [39]. Wie eine klinische Studie mit 53 PACG- und 22 POAG-Patienten ergab, war der mittlere IOD-Tiefstwert bei PACG-Patienten $(12,9 \pm 2,8 \mathrm{mmHg})$ höher als bei POAG-Patienten $(11,5 \pm 3,8 \mathrm{mmHg})$; und der um Mitternacht gemessene mittlere IOD-Wert war bei PACG-Patienten ebenfalls signifikant höher $(14,0 \pm 3,2 \mathrm{mmHg})$ als bei POAG-Patienten $(12,1 \pm 3,7 \mathrm{mmHg})$ [17]. Zudem zeigte eine Studie mit 75 Augen mit PACG nach Iridotomie und 75 gesunden Augen als Kontrollen, dass die kurzzeitigen IOD-Fluktuationen bei PACG-Patienten höher ausfielen $(7,69 \pm 3,03 \mathrm{mmHg})$ als bei gesunden Probanden $(4,83 \pm 2,46$ $\mathrm{mmHg}$ ) [39].

Beim Vergleich von Patientengruppen mit Verdacht auf primären Winkelblock (primary angle closure suspect, PACS), primärem Winkelblock (PAC) und PACG waren die tagsüber auftretenden IOD-Fluktuationen bei Patienten mit PACG und PAC signifikant höher $(5,4 \pm 2,4 \mathrm{mmHg}$ bzw. 4,5 $\pm 2,3 \mathrm{mmHg})$ als bei PACS-Patienten $(3,7 \pm 1,2 \mathrm{mmHg})$ und den gesunden Kontrollen $(3,8 \pm 1,1$ $\mathrm{mmHg}$ ) [73]. Darüber hinaus ergab eine Studie, in der die zirkadianen IOD-Fluktuationen mittels Kontaktlinse in 25 PACG-Augen erfasst wurden, dass Patienten mit Krankheitsprogression gemäß OCT und Gesichtsfelduntersuchung stärkere kurzzeitige IOD-Fluktuationen aufwiesen als Patienten mit stabiler Erkrankung [74]. Größere IOD-Fluktuationen waren bei PACG-Patienten zudem mit dem Ausmaß der Muster-Standardabweichung (pattern standard deviation, PSD) des Gesichtsfelds assoziiert [73]. Die Evidenzlage spricht dafür, dass bei PACG-Patienten eine Korrelation zwischen kurzzeitigen IOD-Fluktuationen und Glaukomprogression besteht.

\section{Okuläre Hypertension (OHT)}

In einer von Grippo et al. durchgeführten Studie mit OHT-Patienten lag der mittlere IOD zwischen $21,1 \mathrm{mmHg}$ und $24,2 \mathrm{mmHg}$ [75]. Sowohl die im Sitzen als auch die in Rückenlage gemessenen IOD-Werte gingen tagsüber kontinuierlich zurück und stiegen nachts an. In dieser Studie fanden sich bei den OHT-Patienten mitternächtliche IOD-Peaks, während die Höchstwerte bei Glaukompatienten und bei den Kontrollgruppen in den frühen Morgenstunden auftraten [75]. Den Ergebnissen einer Studie zufolge betrugen die kurzzeitigen IOD-Fluktuationen 6-8 $\mathrm{mmHg}$, mit einem Höchstwert von $15 \mathrm{mmHg}$ bei OHT $[19,35,37,76]$. In einer anderen Studie, in der die tagsüber gemessenen IOD-Kurven von 690 Patienten analysiert wurden, betrug die tagsüber auftretende IOD-Fluktuation in der OHT-Gruppe 6,8 $\mathrm{mmHg}$ und war damit signifikant höher als in der gesunden Kontrollgruppe $(5 \mathrm{mmHg}$ ) [59].

Zwar fand sich in der Early Manifest Glaucoma Study, der Malmo Ocular Hypertension Study, der Ocular Hypertension Treatment Study sowie einigen anderen Studien kein statistisch signifikanter Zusammenhang zwischen der tagsüber auftretenden IOD-Fluktuation oder Langzeitfluktuation des IODs und der Glaukomprogression [34, 49, 53, 70, 77], doch zeigten sie, dass die Langzeitfluktuation des IODs bei OHT-Patienten, die ein Glaukom entwickelten, höher war $(3,16 \pm 1,35 \mathrm{mmHg})$ als bei Patienten, bei denen dies nicht der Fall war $(2,77 \pm 1,11 \mathrm{mmHg})$ [53]. In einigen ande- ren Studien betrug die tagsüber auftretende IOD-Fluktuation bei Patienten mit Krankheitsprogression $>8 \mathrm{mmHg}$, wohingegen dieser Wert bei Patienten ohne Progression bei $<6 \mathrm{mmHg}$ lag [78]. Dies ist möglicherweise dadurch zu erklären, dass IOD-Fluktuationen positiv mit dem mittleren IOD und dem IOD-Höchstwert korreliert sind [54], die sich in zahlreichen Studien als Risikofaktoren für die Konversion der OHT zum POAG erwiesen haben [19, 78, 79].

In Tabelle 1 sind die 24-Stunden-IOD-Muster bei gesunden Probanden und verschiedenen Glaukomtypen sowie potenzielle prädiktive IOD-Parameter für die Progressionsvorhersage bei verschiedenen Glaukomtypen zusammengefasst.

\section{Therapeutische Wirksamkeit der derzeitigen Therapiemo- dalitäten bei Glaukom in Bezug auf die 24-Stunden-IOD- Muster}

\section{Medikamentöse Therapie}

Die medikamentöse Therapie des Glaukoms umfasst den Einsatz von Prostaglandinanaloga, Betablockern, a2-AdrenozeptorAgonisten, Parasympathomimetika und Carboanhydrasehemmern, meist in Form von Augentropfen [82].

Prostaglandinanaloga (Travoprost, Latanoprost, Bimatoprost, Tafluprost) sind die potentesten topischen antiglaukomatösen Wirkstoffe und in der Lage, eine gleichmäßige 24-stündige IODSenkung um $24-29 \%$ zu erzielen [83-86]. Stewart et al. nahmen eine Metaanalyse von Studien vor, in denen die 24-StundenWirksamkeit von antiglaukomatösen Medikamenten untersucht wurde, und ihren Ergebnisse zufolge waren Bimatoprost gefolgt von Travoprost die beiden Medikamente mit der stärksten IODsenkenden Wirksamkeit $[51,85]$. Es zeigte sich, dass topisches Travoprost $(0,004 \%)$ bei Patienten mit neu diagnostiziertem NTG sowohl den mittleren IOD als auch das IOD-Maximum und -Minimum und die kurzzeitigen IOD-Fluktuationen signifikant reduzierte [87]. Seibold und Kahook wiesen in einer interventionellen Studie mit 27 NTG-Patienten nach, dass Travoprost den IOD zu allen Zeitpunkten während des Tages und in der Nacht signifikant senkte [88]. Eine von Mansouri et al. durchgeführte prospektive klinische Crossover-Studie mit 23 POAG-Patienten ergab, dass Prostaglandinanaloga den IOD-Anstieg beim Übergang vom Wachzustand/Sitzen zum Schlaf/Liegen abflachen können, jedoch keinen Einfluss auf die Akrophase und Amplitude hatten [89]. In einer Crossover-Studie von Orzalesi et al. fand sich eine überlegene Wirksamkeit von Latanoprost in Hinblick auf die IOD-Senkung gegenüber Timolol (Betablocker) zu mehreren Zeitpunkten zwischen 3 Uhr und 21 Uhr und um Mitternacht, und auch gegenüber Dorzolamid (Carboanhydrasehemmer) zu Zeitpunkten zwischen 6 Uhr und 12 Uhr. Das heißt, Latanoprost besaß eine höhere Wirksamkeit und war besser in der Lage, IOD-Fluktuationen zu reduzieren [19, 83, 90]. Gil-Carrasco et al. stellten darüber hinaus fest, dass Prostaglandinanaloga die mittleren IOD-Werte und IOD-Fluktuationen über 24 Stunden bei POAG-Patienten wirksamer senkten als Brinzolamid [91]. Prostaglandinanaloga erwiesen sich zudem als wirksa- 
Tab. 1. Zusammenfassung der 24-Stunden-IOD-Muster bei gesunden Probanden und verschiedenen Glaukomtypen und potenzielle prädiktive IODParameter für die Progressionsvorhersage bei verschiedenen Glaukomtypen

\begin{tabular}{|c|c|c|c|c|c|}
\hline Patientengruppen & $\begin{array}{l}\text { 24-Stunden-IOD- } \\
\text { Rhythmus }\end{array}$ & $\begin{array}{l}\text { Mittlerer IOD } \\
(\mathrm{mmHg})\end{array}$ & $\begin{array}{l}\text { Kurzzeit-IODF } \\
(\mathrm{mmHg})\end{array}$ & $\begin{array}{l}\text { Potenzielle prädiktive } \\
\text { IOD-Parameter zur } \\
\text { Progressionsvorhersage }\end{array}$ & $\begin{array}{l}\text { Sonstige IOD- } \\
\text { Merkmale }\end{array}$ \\
\hline Gesunde Kontrollen & $\begin{array}{l}\text { Druckspitzen beim } \\
\text { Aufwachen [34], Peaks } \\
\text { am Morgen und frühen } \\
\text { Nachmittag, Tiefstwerte } \\
\text { am Nachmittag und } \\
\text { nachts }[6,19,33]\end{array}$ & $14,7 \pm 2,8[32]$ & $4-6[9]$ & - & - \\
\hline $\begin{array}{l}\text { Primäres } \\
\text { Offenwinkelglaukom } \\
\text { (POAG) }\end{array}$ & $\begin{array}{l}\text { Morgens am höchsten } \\
\text { [19, 59], allmählicher } \\
\text { Rückgang über } \\
\text { Tag, am niedrigsten } \\
\text { gegen Mitternacht } \\
\text { [17] mit häufigeren } \\
\text { morgendlichen Peaks } \\
\text { (bes. } 24 \text { Uhr bis } 6 \text { Uhr) [57. }\end{array}$ & $\begin{array}{l}17,6 \pm 3,2[58] \\
19,9 \pm 4,3[57]\end{array}$ & $\begin{array}{l}8,31 \pm 2,58[6,19 \\
39] ; 9,1 \pm 3,6[57]\end{array}$ & $\begin{array}{l}\text { Kurzzeit-IODF und Langzeit- } \\
\text { IODF [19, 33, 43, 44, 51, } \\
\text { 58, 63-65], Anzahl der } \\
\text { langanhaltenden Peaks } \\
{[1,48], \text { mittleres Peak- }} \\
\text { Verhältnis }[1,48]\end{array}$ & $\begin{array}{l}\text { IOD-Peak bei 73,5\% } \\
\text { der Patienten } \\
\text { außerhalb der } \\
\text { Praxiszeiten [57] }\end{array}$ \\
\hline $\begin{array}{l}\text { Sekundäres } \\
\text { Offenwinkelglaukom } \\
\text { (z.B.XFG) }\end{array}$ & $\begin{array}{l}\text { Druckspitze am } \\
\text { Nachmittag }[44,61]\end{array}$ & $20,3 \pm 3,9[60]$ & $\begin{array}{l}\text { Größer als bei } \\
\text { POAG (>15 bei } \\
\text { 35\% der XFG- und } \\
\text { 7,5\% der POAG- } \\
\text { Patienten) }[19,36]\end{array}$ & $\begin{array}{l}\text { Mittlerer IOD [43], } \\
\text { Kurzzeit-IODF [43] }\end{array}$ & $\begin{array}{l}\text { IOD-Peak bei } 45 \% \text { der } \\
\text { unbehandelten XFG- } \\
\text { Patienten außerhalb } \\
\text { der Praxiszeiten [33, } \\
\text { 36], XFG-Patienten } \\
\text { haben höhere } \\
\text { IOD-Höchst- und } \\
\text {-Tiefstwerte als POAG- } \\
\text { Patienten }[19,36,62]\end{array}$ \\
\hline $\begin{array}{l}\text { Normaldruckglaukom } \\
\text { (NTG) }\end{array}$ & $\begin{array}{l}\text { Tagsüber oder in der } \\
\text { Nacht auftretende } \\
\text { Druckspitzen [68,69], } \\
\text { nächtlicher IOD-Peak bei } \\
>50 \% \text { der Patienten [66, } \\
70]\end{array}$ & $\begin{array}{l}11,5 \pm 2,4[66] \\
17,8 \pm 1,6[67]\end{array}$ & $4,4 \pm 1,6[6]$ & $\begin{array}{l}\text { Kurzzeit-IODF }[51,71], \\
\text { Langzeit-IODF }[6,8[54,68], \\
\text { IOD-Minimum }[54,68]\end{array}$ & - \\
\hline $\begin{array}{l}\text { Primäres } \\
\text { Engwinkelglaukom } \\
\text { (PACG) }\end{array}$ & $\begin{array}{l}\text { Morgens am höchsten, } \\
\text { allmählicher Rückgang } \\
\text { über Tag, am niedrigsten } \\
\text { gegen Mitternacht [39] }\end{array}$ & $29,9-45,8[72]$ & $\begin{array}{l}7,69 \pm 3,03 \text { (PACG } \\
\text { nach Iridotomie) } \\
{[39]}\end{array}$ & Kurzzeit-IODF $[73,74]$ & $\begin{array}{l}\text { Der mittlere IOD- } \\
\text { Tiefstwert und } \\
\text { die mittleren } \\
\text { mitternächtlichen IOD- } \\
\text { Werte sind bei PACG } \\
\text { höher als bei POAG } \\
\text { [17], Ausmaß der } \\
\text { tagsüber auftretenden } \\
\text { IOD-Fluktuation: } \\
\text { PACG > PAC > PACS [73] }\end{array}$ \\
\hline $\begin{array}{l}\text { Okuläre Hypertension } \\
(\mathrm{OHT})\end{array}$ & $\begin{array}{l}\text { Rückgang über Tag und } \\
\text { Anstieg in der Nacht mit } \\
\text { mitternächtlichen Peaks } \\
\text { [75] }\end{array}$ & $21,1-24,2[75]$ & $\begin{array}{l}6-8[19,35,37,76] ; \\
6,8[59]\end{array}$ & $\begin{array}{l}\text { Mittlerer IOD [54, 78, 79, 81], } \\
\text { IOD-Peak [54, 78, 79, 81], } \\
\text { Kurzzeit-IODF [78], Langzeit- } \\
\text { IODF [53] }\end{array}$ & - \\
\hline
\end{tabular}

IOD: Augeninnendruck; IODF: IOD-Fluktuation; XFG: exfoliatives Glaukom.

mer zur Verringerung der 24-Stunden-IOD-Fluktuationen bei Patienten mit POAG und NTG als die selektive Laser-Trabekuloplastik (SLT) [70]. Die abendliche Anwendung von Latanoprost oder der Latanoprost-Timolol-Kombinationstherapie senkte den IOD am Tag bei POAG-Patienten stärker als die morgendliche Anwendung [19, 81, 83, 92]. In einer prospektiven placebokont- rollierten Studie von Konstas et al. zeigte darüber hinaus die abendliche Anwendung der Tafluprost-Timolol-Fixkombination eine höhere 24-stündige Wirksamkeit bei der Verbesserung des IOD-Profils von POAG-Patienten als die morgendliche Anwendung [93]. Eine kontrollierte Crossover-Studie mit 44 Patienten mit POAG oder OHT fand keinen statistisch signifikanten Un- 
terschied in Hinblick auf die 24-Stunden-Wirksamkeit von Latanoprost, Travoprost und Bimatoprost. Allerdings liegen Hinweise vor, denen zufolge Travoprost und Bimatoprost möglicherweise eine gleichmäßigere IOD-Senkung über 24 Stunden bieten als Latanoprost [83, 85, 94-97].

In einer Metaanalyse erreichte 0,5\%ige Timololol-Lösung eine mittlere zirkadiane IOD-Senkung von $19-24 \%$ gegenüber dem unbehandelten Ausgangszustand, und dies trotz reduzierter nächtlicher Wirksamkeit $[83,85]$. Seibold et al. führten eine Studie mit 60 Patienten mit OAG oder OHT durch und zeigten, dass 0,5\%ige Timololol-Lösung in der Lage war, den IOD am Tag zu senken, nicht jedoch den nächtlichen IOD [98]. Neben der 0,5\%igen Timololol-Lösung besaß nach Berichten von Quaranta et al. Timogel 0,1\% (Timololol 0,1\% in gelbildenden Carbomeren) eine ähnliche Wirksamkeit wie die Lösung in Hinblick auf die Senkung des mittleren IODs über 24 Stunden, der Werte am Tag und in der Nacht und zu verschiedenen Einzelzeitpunkten [99]. Die Anwendung von Timolol in einer Carbomer-Gelform ist in der Lage, eine vergleichbare Kontrolle des IOD-Profils zu erzielen, jedoch bei geringerer Konzentration, wodurch die Symptome, die denen des trockenen Auges ähneln, reduziert werden und das Medikament besser verträglich wird [100, 101]. Bei dreimal täglicher Anwendung bewirkte Dorzolamid eine Senkung des zirkadianen IODs um 15 bis $23 \%$ [83, 85, 90, 102]. Nach Orzalesi et al. waren Timolol und Dorzolamid ähnlich wirksam, doch zeigte Dorzolamid eine signifikant bessere nächtliche Wirksamkeit als Timolol [83, 90]. Brimonidin (ein selektiver a2-AdrenozeptorAgonist) senkte bei zweimal täglicher Anwendung ebenfalls den mittleren IOD über 24 Stunden um 17,3\%, besaß jedoch keine nächtliche Wirksamkeit [83, 103]. Liu et al. stellten fest, dass Wirkstoffe, die die Kammerwasserproduktion vermindern (darunter ein Betablocker und ein selektiver a2-AdrenozeptorAgonist) bei einem niedrigeren nächtlichen IOD wenig wirksam sind, weil die Kammerwasserproduktion im Schlaf bereits um $50 \%$ reduziert ist, was die beschriebene geringe nächtliche Wirksamkeit beider Medikamente erklärt [43, 104, 105].

Eine Metaanalyse untersuchte die Wirksamkeit von Kombinationstherapien zur Senkung des mittleren IODs am Tag. Dabei fielen die relativen Verringerungen unter der Kombination aus Betablocker und Prostaglandinanaloga höher aus (34,9\% für die Fixkombination Travoprost/Timolol, 34,3\% für Bimatoprost/ Timolol und 33,9\% für Latanoprost/Timolol) als unter der Kombination aus einem Carboanhydrasehemmer oder a2-Agonisten und einem Betablocker (29,9\% für die Fixkombination Dorzolamid/Timolol und 28,1\% für Brimonidin/Timolol) [19, 106]. Im Vergleich zur Latanoprost- oder Timolol-Monotherapie bewirkte die Latanoprost-Timolol-Fixkombination außerdem einen stärkeren Rückgang des IODs zu jedem Zeitpunkt der 24-StundenKurve und eine stärkere Abnahme der IOD-Fluktuationen [19, $107,108]$.

\section{Chirurgische Therapie}

$\mathrm{Zu}$ den gängigen Operationstechniken bei Glaukom gehören die Laser-Trabekuloplastik und die Trabekulektomie (auch als Filtra- tionschirurgie bezeichnet). Lee et al. untersuchten die Wirksamkeit der Laser-Trabekuloplastik bei 28 Augen von 18 behandelten Glaukompatienten und berichteten, dass die Laserbehandlung eine gleichmäßigere Senkung des nächtlichen IODs bewirkte [51, 109], jedoch nicht zu einem signifikanten Rückgang des mittleren IODs, der IOD-Peaks oder des tagsüber gemessenen IODs führte $[33,109,110]$. Wie bereits beschrieben, weisen NTG-Patienten in der Regel tagsüber auftretende oder nächtliche IOD-Peaks [69] auf, und bei $60 \%$ der Patienten findet sich der Peak in der Nacht [40]. Die Laser-Trabekuloplastik verbessert den in der Nacht deutlich reduzierten Kammerwasserabfluss und bewirkt so eine deutliche Verringerung der nächtlichen IOD-Fluktuationen [43]. Dies könnte von Bedeutung sein für die Verhinderung der NTGProgression, obgleich sich die Spanne der 24-Stunden-IOD-Fluktuationen und der tagsüber auftretenden IOD-Fluktuationen nach der Laser-Trabekuloplastik nicht signifikant ändert [111]. Kóthy et al. zeigten in einer klinischen Studie mit 26 Augen von 13 Patienten, dass die selektive Laser-Trabekuloplastik auch bei Patienten mit POAG hauptsächlich die Amplitude der tagsüber auftretenden IOD-Fluktuationen verringert, jedoch keine signifikanten Rückgang des mittleren IODs bewirkt [112].

Die Trabekulektomie war besonders wirksam bei der Verringerung der tagsüber auftretenden IOD-Fluktuationen bei Patienten mit POAG $[15,113]$ und PACG [114] und sie verlangsamte die Krankheitsprogression $[34,53,114]$. In einer Studie von Medeiros et al., in der die Trabekulektomie mit der medikamentösen Therapie bei Glaukompatienten verglichen wurde, nahm die postoperative kurzzeitige IOD-Fluktuation auf 2,3 $\pm 0,8 \mathrm{mmHg}$ ab, während sie in der medikamentös behandelten Gruppe bei 4,8 $\pm 2,3 \mathrm{mmHg}$ lag $[19,39,115]$. Eine prospektive Beobachtungsstudie von Mansouri et al. mit 60 Patienten ergab, dass der mittlere IOD am Tag bei den mit Trabekulektomie behandelten Patienten signifikant niedriger war als der von Patienten, die eine Latanoprost-Monotherapie oder tiefe Sklerektomie mit Kollagenimplantat erhalten hatten [33, 110]. Konstas et al. verglichen in einer Studie Patienten mit fortgeschrittenem OAG, bei denen eine Trabekulektomie durchgeführt worden war, und Patienten unter medikamentöser Maximaltherapie. Eine andere Studie von Ross et al. zeigte ebenfalls, dass eine erfolgreiche Trabekulektomie wirksamer war, wenn es darum ging, den mittleren IOD, den IOD-Peak und die IOD-Fluktuationen über 24Stunden zu verringern $[33,116,117]$. Eine Fallstudie von Park et al. ergab, dass die Trabekulektomie bei JOAG die tagsüber auftretenden IOD-Fluktuationen wirksamer verringerte als eine optimale medikamentöse Therapie [11].

In Tabelle 2 ist die therapeutische Wirksamkeit der derzeitigen Therapiemodalitäten zur Glaukombehandlung in Bezug auf die 24-Stunden-IOD-Muster zusammengefasst.

\section{Diskussion}

In der aktuellen klinischen Praxis werden die intraokularen Druckprofile der Patienten hauptsächlich durch punktuelle IODMessungen in der Praxis mithilfe der GAT, Applanationstonometrie mittels Handgerät und Pneumotonometrie geschätzt. Im 
Tab. 2. Therapeutische Wirksamkeit der derzeitigen Therapiemodalitäten zur Glaukombehandlung in Bezug auf die 24-Stunden-IOD-Muster.

\begin{tabular}{ll}
\hline Therapiemodalitäten & Therapeutische Wirksamkeit in Bezug auf die 24-Stunden-IOD-Muster \\
\hline Prostaglandinanaloga (z.B. Travoprost, Latanoprost, & (1) Erreichen eine gleichmäßige 24-Stunden-IOD-Senkung von 24-29\% [83-86] \\
Bimatoprost, Tafluprost) & (2) Senkung des mittleren IODs, des IOD-Maximums und -Minimums und der kurzzeiti- \\
gen IODF bei NTG [87] & (3) Abflachung des IOD-Anstiegs beim Übergang vom Wachzustand/Sitzen zum \\
Schlaf/Liegen bei POAG [89] & (4) Die abendliche Anwendung senkte bei POAG den IOD am Tag besser als die mor- \\
gendliche Anwendung [19, 81, 83, 92] & (5) Wirksamer als Betablocker und Carboanhydrasehemmer zur Senkung des IODs zu \\
mehreren Zeitpunkten und zur Senkung des mittleren IODs und der IODF [19, 83, 90, & 91] \\
(6) Die stärksten IOD-senkenden Medikamente waren Bimatoprost gefolgt von Travo- & prost [51, 85] \\
(7) Wirksamer als die selektive Laser-Trabekuloplastik zur Senkung der 24-Stunden-IODF & bei POAG und NTG [70] \\
\hline
\end{tabular}

Betablocker (z.B. Timolol)

(1) Erreichen eine mittlere 24-Stunden-IOD-Senkung von 19-24\% [83, 85]

(2) Geringe Wirksamkeit bei der Senkung des nächtlichen IODs [83, 85, 98]

(3) 0,5\%ige Timolol-Lösung und Timogel 0,1\% zeigten eine ähnliche Wirksamkeit bei der Senkung des mittleren IODs über 24 Stunden, der Tages- und Nachtwerte und der Werte zu verschiedenen Einzelzeitpunkten [99], und Timogel 0,1\% war besser verträglich $[100,101]$

$\begin{array}{ll}\text { Carboanhydrasehemmer (z.B. Dorzolamid) } & \text { (1) Erreichen eine mittlere 24-Stunden-IOD-Senkung von 15-23\% [83, 85, 90, 102] } \\ \text { (2) Überlegene nächtliche Wirksamkeit im Vergleich zu Timolol [83, 90] }\end{array}$

a2-Adrenozeptor-Agonisten (z.B. Brimonidin)

(1) Erreichen eine mittlere 24-Stunden-IOD-Senkung von 17,3\% [83, 103]

(2) Geringe Wirksamkeit bei der Senkung des nächtlichen IOD [83, 103]

Kombinationspräparate

(1) Die Kombination von Prostaglandinanaloga und Betablockern war wirksamer als die Kombination eines Carboanhydrasehemmers oder eines a2-Agonisten und Betablockern zur Senkung des mittleren IOD am Tag $[19,106]$

(2) Die Fixkombination von Prostaglandinanaloga und Betablockern senkt den IOD stärker als die jeweilige Monotherapie [19, 107, 108]

(3) Bei Patienten mit POAG war die abendliche Anwendung der Prostaglandin-Timo-

lol-Fixkombination im Hinblick auf die 24-Stunden-IOD-Kontrolle gegenüber der morgendlichen Anwendung überlegen $[19,81,83,92,93]$

Laser-Trabekuloplastik

(1) Wirksame IOD- und IODF-Senkung während der Nachtstunden [33, 109, 110]

(2) Keine signifikante Senkung des mittleren IOD, des IOD-Peaks und des IOD am Tag $[68,111]$

Trabekulektomie

(1) Wirksame IODF-Senkung am Tag bei POAG und PACG [15, 113, 114, 118]

(2) Erreicht geringere kurzzeitige IODF sowie einen niedrigeren mittleren IOD am Tag

und IOD-Peak als die medikamentöse Therapie $[45,68,71,74]$

IOD: Augeninnendruck; IODF: IOD-Fluktuation; POAG: primäres Offenwinkelglaukom; NTG: Normaldruckglaukom

Hinblick auf die IOD-Überwachung und Beurteilung der Glaukomprogression ist es interessant, dass es Menschen mit normalen IOD-Werten oder zufriedenstellender IOD-Kontrolle gibt [2, 9-12], die dennoch eine Gesichtsfeldverschlechterung und sogar irreversible Blindheit entwickeln. Neuere Studien sprechen dafür, dass neben den punktuellen IOD-Messungen in der Praxis auch die Peaks, der Verlauf und die Fluktuationen des IODs eine Rolle bei der Glaukomprogression spielen $[1,40,42,47,48]$, wobei einige Studien allerdings zu anderen Ergebnissen kamen [19, 49, 50, 52-55]. Nach unserer Auffassung ist diese Diskrepanz durch das unterschiedliche Studiendesign und die Vielzahl der GlaukomSubtypen zu erklären. Daher nahmen wir eine kritische Überprü- fung der Literatur vor und erstellten eine Zusammenfassung der wichtigen Studienergebnisse zu den 24-Stunden-IOD-Mustern bei primärem und sekundären Offenwinkelglaukom, NTG, PACG und OHT.

Der mittlere IOD, der sich auch durch die Mittelung der punktuellen IOD-Messungen in der Praxis abschätzen lässt und die kurzzeitigen IOD-Fluktuationen waren bei Patienten mit POAG $[6,19$, $39,57,58]$, sekundärem Offenwinkelglaukom (z.B. XFG) $[19,36$, 60], PACG [39, 72] und OHT [19, 35, 37, 59, 75, 76] höher als bei gesunden Probanden $[6,19,32]$, nicht jedoch bei Patienten mit NTG $[6,66]$. Der zirkadiane IOD-Rhythmus bei POAG war allgemein ähnlich wie bei gesunden Probanden, in dem Sinne, dass der 
IOD morgens am höchsten war und im Laufe des Tages allmählich abnahm $[6,17,19,33,34,59]$. Allerdings kam es bei POAG häufiger zu morgendlichen Peaks und der IOD-Peak trat bei 73,5\% der Patienten außerhalb der Praxiszeiten auf [57]. Im Vergleich zum primären Offenwinkelglaukom zeigte das sekundäre Offenwinkelglaukom (z.B. XFG) ein anderes zirkadianes Muster mit stärkeren Schwankungen $[19,36]$. Dabei kam es meist zu nachmittäglichen Peaks [44, 61], und der IOD-Peak war daher nicht immer leicht während der Praxiszeiten zu erfassen [33, 36]. Da sich die Kurzund Langzeit-IOD-Fluktuationen [19, 33, 43, 44, 51, 58, 63-65] sowie die Anzahl der langanhaltenden Peaks $[1,48]$ und das mittlere Peak-Verhältnis $[1,48]$ ebenfalls als prädiktive Faktoren für die Progressionsvorhersage bei primärem und sekundärem Offenwinkelglaukom erwiesen haben, kann die 24-Stunden-IOD-Messung Ärzten in klinischen Settings, in denen Patienten eine Gesichtsfeldverschlechterung, jedoch zufriedenstellende punktuell in der Praxis gemessene IOD-Werte haben, helfen, morgendliche und nachmittägliche Peaks und IOD-Fluktuationen zu erkennen, die mögliche Therapieänderungen zur Folge haben können. Bei NTG-Patienten ist die 24-Stunden-IOD-Messung unter Umständen besonders wichtig, da der gemessene mittlere IOD im Allgemeinen ähnlich ausfällt wie bei gesunden Probanden, so dass nicht viele Anhaltspunkte für die Diagnose und anschließende Überwachung der Krankheitsprogression vorlägen. NTG-Patienten hatten typischerweise vor allem am Tag und in der Nacht auftretende IOD-Druckspitzen $[68,69]$ und bei bis zu $60 \%$ von ihnen traten die höchsten IOD-Druckspitzen um Mitternacht auf [66, 70]. Es ist zu beachten, dass sich neben dem mittleren IOD [54, 68] auch die Kurzzeit- $[51,71]$ und Langzeitfluktuationen des IODs [6, 80] sowie der IOD-Tiefstwert $[54,68]$ als prädiktive Parameter für die Progressionsvorhersage bei NTG erwiesen haben. Dies spricht dafür, dass bei Patienten mit NTG zur Vorhersage des Progressionsrisikos möglicherweise eine 24-Stunden-IOD-Messung erfolgen muss, die zudem als Orientierungshilfe für die Diagnosestellung und individuelles Therapie dient. Patienten mit PACG wiesen einen ähnlichen 24-Stunden-IOD-Rhythmus auf wie gesunde Probanden [39], wohingegen OHT-Patienten ein invertiertes zirkadianes Muster zeigten, bei dem der IOD im Tagesverlauf abnahm und in den Nachtstunden anstieg [75]. Kurzzeitige IODFluktuationen [73, 74, 78], Langzeitfluktuationen [53] und IODPeaks [54, 78, 79, 81], die mittels 24-Stunden-IOD-Messung bei PACG und OHT festgestellt werden, können als Ergänzung zu den punktuellen Messungen in der Praxis hilfreich sein, um Schäden am Sehnervenkopf und einen Gesichtsfeldverlust, d. h. die Entwicklung von einem Verdacht auf primären Winkelblock oder OHT zu einem PACG oder POAG vorherzusagen.

Vor dem Hintergrund, dass vorgeschlagen wurde, ein umfassenderes Behandlungsziel für die 24-Stunden-IOD-Profile der Patienten festzulegen, haben wir zudem die Wirksamkeit von antiglaukomatösen Arzneimitteln, Laser-Trabekuloplastik und Trabekulektomie zur Verbesserung der verschiedenen 24-Stunden-IOD-Parameter erörtert. Aktuellen Daten zufolge waren Prostaglandinanaloga (insbesondere Bimatoprost und Travoprost $[51,85]$ ) die wirksamsten topischen Augentropfen, um eine gleichmäßige Senkung des IODs über 24 Stunden zu erreichen [83-86], und sie bewirkten darüber hinaus eine Verringerung der IOD-Höchst- und Tiefstwerte und der kurzzeitigen IOD-Fluktuationen [87]. Insbesondere senkte die abendliche Anwendung von Prostaglandinanaloga bei POAG den IOD am Tag besser als die morgendliche Anwendung [19, 81, 83, 92]. Alpha2-Adrenozeptor-Agonisten und Betablocker wiesen eine vergleichbare IOD-senkende Wirkung, jedoch eine geringere nächtliche Wirksamkeit auf [83, 85, 98, 103], während Carboanhydrasehemmer demgegenüber eineüberlegenenächtliche Wirksamkeit zeigten $[43,83]$. Unter allen Arten von Arzneimittelkombinationen waren die Fixkombinationen von Prostaglandinanaloga und Betablockern die effektivsten bei der Verbesserung des IOD-Musters über 24 Stunden $[19,106]$. Daher wird Ärzten empfohlen, die unterschiedlichen antiglaukomatösen Monotherapien oder Kombinationstherapien entsprechend dem jeweiligen zirkadianen Rhythmus der einzelnen Glaukom-Subtypen oder gemäß dem individuellen IOD-Profil auszuwählen. Allerdings ist zu beachten, dass einige antiglaukomatöse Medikamente wie z. B. Timolol [119] und Apraclonidin [120, 121] in einigen wenigen Studien bei Langzeitanwendung mit Tachyphylaxie assoziiert waren, und ihre langfristige klinische Bedeutung in Hinblick auf die 24-Stunden-IOD-Muster näher untersucht werden muss. Was die chirurgischen Optionen betrifft, führte die selektive Laser-Trabekuloplastik zwar nicht zu einer Veränderung des IOD-Gesamtmusters über 24 Stunden, doch war sie in der Lage, die nächtlichen IODDruckspitzen zu verringern [43], die zur NTG-Progression beitragen können [111]. Es sei jedoch darauf hingewiesen, dass bei POAG- und NTG-Patienten Prostaglandinanaloga die kurzzeitigen IOD-Fluktuationen, die ein prädiktiver Faktor für die Krankheitsprogression waren, wirksamer verringerten als die LaserTrabekuloplastik [70]. In Hinblick auf die Verringerung der tagsüber auftretenden IOD-Fluktuationen bei Patienten mit POAG $[15,113]$ und PACG $[114]$ war dagegen die Trabekulektomie wirksamer als die medikamentöse Therapie $[45,68,71,74]$ und sie verlangsamte die Krankheitsprogression [34, 53, 114].

Derzeit verfügbare Verfahren zur Durchführung der 24-Stunden-IOD-Messung in der klinischen Praxis sind unter anderem die Selbsttonometrie mit einem tragbaren Tonometer, die permanente IOD-Messung mithilfe eines implantierten IOD-Sensors und ein 24-Stunden-IOD-Monitoring-System wie der SENSIMED Triggerfish ${ }^{\circledR}$ Einweg-Kontaktlinsen-Sensor (CLS). Das CLS-System empfiehlt sich vor allem, weil es nicht invasiv, leicht zu entfernen [23], allgemein verfügbar [23] sowie gut verträglich $[20,27,28]$ ist und zudem eine hohe Reproduzierbarkeit besitzt [28-30]. Allerdings müssen die Validität (d h. die Genauigkeit der IOD-Schätzung) [29, 30] des CLS-Systems bei Anwendung über 24 Stunden hinaus und seine Kosteneffizienz in der Glaukomtherapie noch untersucht werden. Nach Schätzungen des britischen National Institute for Health and Care Excellence kostet die 24-stündige Anwendung des SENSIMED Triggerfish ${ }^{\circledR}$ CLS-Systems zwischen 526 und 682 US-Dollar, zusätzlich zu den Kosten von rund 7310 US-Dollar für den wiederverwendbaren Datenrekorder, Kabel und Software [20]. 


\section{Schlussfolgerung}

Nach Überprüfung der aktuellen Literatur kamen wir zu dem Schluss, dass die 24-Stunden-IOD-Messung eine wertvolle Ergänzung zu den punktuellen IOD-Messungen in der Praxis sein kann, da sie Ärzten Informationen über den zirkadianen Gesamtrhythmus des IODs, den zeitlichen Verlauf von IOD-Peaks und das Ausmaß der IOD-Fluktuationen bei den unterschiedlichen Glaukom-Subtypen und verschiedenen Patienten liefert. Verschiedene IOD-Parameter, darunter der mittlere IOD, Kurz- und Langzeitfluktuationen des IODs sowie die IOD-Tiefst- und Höchstwerte erwiesen sich in mehreren Studien als prädiktiv für die Glaukomprogression. Möglicherweise spielen diese Parameter eine wichtige Rolle für die Früherkennung und zukünftige Diagnose des NTG und für die Verlaufskontrolle bei Patienten, bei denen es trotz zufriedenstellender, punktuell in der Praxis gemessener IOD-Werte zu Gesichtsfeldverschlechterungen kommt. Es ist zu betonen, dass trotz der scheinbar vielversprechenden klinischen Bedeutung des 24-Stunden-IOD-Monitorings die verfügbaren Daten aus der aktuellen Literatur in Bezug auf Patientenmerkmale, Einschlusskriterien, Verfahren der 24-StundenIOD-Messung und Studienmethoden, die die klinische Anwendung der 24-Stunden-IOD-Messung stützen sollen, nach wie vor uneinheitlich sind. Groß angelegte standardisierte prospektive Studien wären unbedingt erforderlich, um - wie erörtert - den Zusammenhang der 24-Stunden-IOD-Muster mit der Glaukomprogression bei den verschiedenen Glaukom-Subtypen eingehender zu untersuchen und die Wirksamkeit der unter- schiedlichen Therapiemodalitäten zur Verbesserung der IODMuster zu bewerten. Zudem sollten auch die Validität, Kosteneffizienz und Nebenwirkungsprofile der verschiedenen Modalitäten der 24-Stunden-IOD-Messung näher untersucht werden. Im Übrigen werden Kliniker vor dem Hintergrund zunehmend vorliegender wissenschaftlicher und klinischer Belege ermutigt, die Behandlung auf die schlecht eingestellten IOD-Parameter auszurichten, die bei Patienten mit unverhältnismäßig starker Glaukomprogression im Verhältnis zu den in der Praxis gemessenen IOD-Werten identifiziert wurden.

\section{Interessenkonflikte}

Keiner der Autoren hat Interessenkonflikte offenzulegen.

\section{Literatur}

Die Literatur ist als Supplemental Material unter www.karger. com/?=DOI505890 verfügbar.

\section{Lizenzangabe}

Chun Hing Ho, Jasper K. W. Wong: Role of 24-hour intraocular pressure monitoring in glaucoma management. J Ophthalmol 2019;2019:3632197 (https:// doi.org/10.1155/2019/3632197), ${ }^{\circledR} 2019$ Chun Hing Ho and Jasper K. W. Wong (Übersetzung, «Abbrevations» gekürzt), lizensiert unter CC BY 4.0 (https:// creativecommons.org/licenses/by/4.0/deed/de). 